\title{
Positional order in Langmuir monolayers: An x-ray diffraction study
}

\author{
V. M. Kaganer,* G. Brezesinski, and H. Möhwald \\ Max-Planck-Institute of Colloids and Interfaces, D-12489 Berlin, Germany \\ P. B. Howes and K. Kjaer \\ Physics Department, Ris N National Laboratory, DK-4000 Roskilde, Denmark
}

(Received 16 June 1998)

\begin{abstract}
The structural phase transition from the hexagonal to a distorted-hexagonal (centered rectangular) phase (the $L S-S$ transition) in Langmuir monolayers of octadecanol is studied in a grazing incidence x-ray diffraction experiment. We find algebraic decay of positional correlations, which suggests solid-like order in both phases at the distances accessible in the present $\mathrm{x}$-ray diffraction experiment. The transition is accompanied by strong positional disorder, which is evident from the drastic increase of the exponent $\eta$ to values $\eta>2$ close to the transition. Remarkably, on approaching the transition, the continuous increase of $\eta$ is apparent already at $20^{\circ} \mathrm{C}$ above it. The positional disorder is attributed to elastic distortions around pretransitional fluctuations. [S1063-651X(99)10102-8]
\end{abstract}

PACS number(s): 68.10.-m, 68.18.+p, 68.35.Rh, 64.70.Md

\section{INTRODUCTION}

The bulk of the understanding of positional order in twodimensional systems comes from theoretical studies of systems consisting of circularly symmetric particles that do not possess internal degrees of freedom. In experimental studies it is frequently assumed that these degrees of freedom are not relevant for the positional ordering. It is well known that in a two-dimensional solid the low dimensionality of the system gives rise to an algebraic decay of positional correlations at any nonzero temperature [1]. On increasing the temperature the solid can either melt directly into a two-dimensional liquid or form an intermediate hexatic phase, with short-range positional order and quasi-long-range orientational order [2].

Langmuir monolayers (insoluble monolayers on a liquid surface) exhibit phase transitions due to coupled orientational degrees of freedom of the individual molecules and their positions [3]. Numerous studies of Langmuir monolayers by means of grazing-incidence $\mathrm{x}$-ray diffraction have primarily utilized the horizontal peak positions to determine the lattice and the (vertical) Bragg rod intensity profiles for microscopic structural information [4-6]. The information on positional correlations, which is contained in the horizontal profiles of the diffraction peaks, has remained unused.

The aim of the present paper is to obtain the correlation functions of positional order in different phases of Langmuir monolayers directly by processing the experimental data, without any presupposed model, and then analyze the correlation functions themselves, rather than the peak profiles. As discussed in detail below, this is possible because the monolayer is a two-dimensional powder of randomly oriented domains. The average of the structure factor over the orientations of the domains is equivalent to its integration over the transverse component of the scattering vector. Transforming to real space, this integration singles out the correlation func-

\footnotetext{
*Permanent address: Institute of Crystallography, Russian Academy of Sciences, 117333 Moscow, Russia.
}

tion in the direction of the reciprocal-lattice vector.

This study has concentrated on two condensed phases of Langmuir monolayers of octadecanol on water. The hightemperature phase, LS, possesses a single first-order diffraction peak due to hexagonal local arrangement of the molecules. The lower-temperature phase, S, reveals two firstorder peaks of the centered rectangular unit cell due to ordering of short axes of the molecules. In both phases, the molecules are not tilted with respect to the surface normal. We have found that, on the available length scale limited by the resolution, the correlation function $G(x)$ exhibits algebraic decay, $G(x) \propto x^{-\eta}$. The exponent $\eta$ depends on temperature and varies from $\eta \approx 0.5$ far from the $L S-S$ phase transition to about 3 close to it. There is no evidence for a characteristic length (e.g., a "crystallite size") that would limit the algebraic decay at large $x$. However, the algebraic decay is limited on the small $x$ side at a characteristic length $L$ of some tens of Angströms, which also depends on temperature and increases at the phase transition. Even the smallest values of the exponent $\eta$ found in the present study exceed the limit $\eta<1 / 3$, which can be reached in the solid phase before the dislocation-mediated melting transition according to Ref. [2]. One can suggest [7] that the large exponent is realized because of a large core energy of the dislocations that prevents the dislocation mediated melting. Then the shear modulus of the two-dimensional solid can be derived from the value of $\eta$ and is small compared with the compression modulus. However, the increase of the exponent at the transition $L S-S$ points to another source of positional disorder in the monolayer (in addition to the longwavelength phonon fluctuations), related to the distortion of the unit cell from hexagonal to distorted hexagonal (centered rectangular).

We consider orientational fluctuations of the molecules close to the transition $L S-S$ and positional fluctuations coupled to them assuming that the effects of unbinding of dislocation pairs are not relevant to the problem either because the temperature is small in comparison with the transition temperature for dislocation-mediated melting or be- 


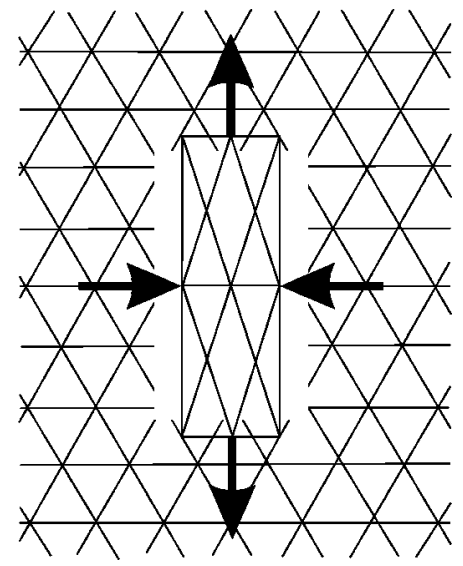

FIG. 1. A centered rectangular (distorted hexagonal) cluster in the hexagonal matrix and the strains it causes in the matrix.

cause the dislocation core energies are large. The ordering of the short axes (molecular backbones) at the $L S-S$ transition is accompanied by a large $(\approx 9 \%)$ rectangular distortion of the initially hexagonal unit cell and some $(\approx 1.5 \%)$ change in the molecular area. The fluctuations with the structure of the backbone-ordered phase cause elastic distortions in the surrounding matrix, Fig. 1. These distortions, in the same way as elastic distortions caused by localized defects of any nature, give rise to an additional contribution to the exponent $\eta$. The value of this contribution is not restricted by the dislocation melting theory; it increases close to the $L S-S$ transition point due to increasing fluctuations. The length $L$ limiting the algebraic decay at small distances is proportional to the correlation length of the fluctuations; it also increases close to the transition. Analogously, fluctuating disorder close to the transition point below it explains the temperature variation of the parameters $\eta$ and $L$ in the backbone-ordered phase $S$.

\section{EXPERIMENT}

The study was performed mainly on octadecanol $\mathrm{CH}_{3}\left(\mathrm{CH}_{2}\right)_{17} \mathrm{OH}$. Some additional measurements were done with hexadecanol $\mathrm{CH}_{3}\left(\mathrm{CH}_{2}\right)_{15} \mathrm{OH}$ and tetradecanol $\mathrm{CH}_{3}\left(\mathrm{CH}_{2}\right)_{13} \mathrm{OH}$. All substances (Fluka, Germany, purity $>99 \%$ ) were used without further purification.

The phase diagram of the octadecanol monolayers is well established [8-11]. The diffraction measurements were performed mostly in the untilted phases at a surface pressure of $18 \mathrm{mN} / \mathrm{m}$, well above the tilting phase transition that occurs, depending on temperature, between 10 and $14 \mathrm{mN} / \mathrm{m}$. X-ray diffraction studies of the tilted phases of octadecanol were reported earlier $[12,13]$. The isotherms monitored during the diffraction experiment reproduce the order and the surface pressure of the tilting phase transition reported in Refs. [8-11]. Other phase transitions found in the isotherm study [10] were not seen. Also, our previous x-ray diffraction study [12] did not reveal structural changes corresponding to these reported transitions.

The grazing incidence $\mathrm{x}$-ray diffraction measurements were performed at the liquid surface diffractometer on the undulator beamline BW1 at HASYLAB, DESY, Hamburg. A beam of wavelength $1.304 \AA$ was incident on the surface at a shallow angle $\alpha=0.85 \alpha_{c}$ (where $\alpha_{c}$ is the critical angle for total external reflection), to reduce the background scattering from the water subphase. The footprint of the beam on the water surface was $5 \times 50 \mathrm{~mm}^{2}$. The horizontal resolution was measured by scanning through the beam specularly reflected from the water surface. It is given mainly by a Soller collimator placed in front of a vertical positionsensitive detector (PSD). Smaller contributions to the horizontal resolution come from the angular divergence of the white synchrotron beam and from the small mosaic width of the Beryllium monochromator crystal [14]. The shape of the resolution function was found to be very close to a Gaussian with a full width at half-maximum, $\mathcal{W}\left(Q_{x y}\right)=0.0082 \AA^{-1}$, giving rise to a spatial resolution $2 \pi / \mathcal{W} \approx 770 \AA$. The vertical resolution, given by the PSD, is $\mathcal{W}\left(Q_{z}\right)=0.005 \AA^{-1}$.

All peaks (except for the measurements performed in the tilted phase at a low surface pressure of $1 \mathrm{mN} / \mathrm{m}$ ) lie in the water plane, $Q_{z} \simeq 0$. The intensity was summed over the channels of the PSD, since the present study concerns the in-plane order in the monolayer.

\section{DIFFRACTION PEAKS AND THEIR ANALYSIS}

\section{A. Structure factors and correlation functions in the powder diffraction experiment}

It is well established that the diffraction patterns of Langmuir monolayers are due to a two-dimensional powder of randomly oriented domains. In the optically anisotropic phases, the size of well-oriented domains can be directly determined from Brewster angle microscopy observations [15] and is in a range of tens to hundreds of microns. The number of domains in the beam footprint $\left(5 \times 50 \mathrm{~mm}^{2}\right)$ is thus sufficiently large. Taking into account that the tilt azimuth is fixed with respect to the lattice at either the nearestneighbor or the next-nearest-neighbor (NNN) direction, one concludes that in the tilted phases the lattice orientation only slightly varies within a domain. One can expect that the domains of constant lattice orientation are much larger than the resolution limit of the $\mathrm{x}$-ray diffraction experiment in the untilted phases as well. Hence, we consider scattering from a 2D powder of randomly oriented domains and neglect the finite sizes of the domains. The latter assumption is confirmed by the correlation functions obtained which do not show any cutoff length.

The considerations below are based on the well-known approach by Warren and Averbach [16] who treated the powder diffraction peak profiles in terms of the real-space quantities and used Fourier transformation to deconvolve intrinsic peak profiles from resolution effects. However, the original treatment [16], as well as its further development (see, e.g., [17-19]), was given in terms of finite sizes of crystallites and mean strains in them. The concept of the correlation function is not used in the field of powder diffraction and we are not aware of the explicit representation, in the powder-diffraction literature, of the structure factor of the powder-diffraction experiment in terms of the correlation function, as given below.

The amplitude of the $\mathrm{x}$-ray scattering from any set of atoms can be represented as a sum, 


$$
A(\mathbf{Q})=\sum_{j} f_{j}(\mathbf{Q}) \exp \left(i \mathbf{Q} \cdot \mathbf{R}_{j}\right),
$$

where $\mathbf{Q}$ is the scattering vector (the difference between the wave vectors of the scattered and the incident waves), $\mathbf{R}_{j}$ is the position of the $j$ th atom, $f_{j}(\mathbf{Q})$ is its scattering factor, and the sum runs over all atoms. In the condensed phases of Langmuir monolayers, the molecules can be represented, in the simplest approximation, as rigid rods oriented parallel to each other. Then the summation over all atoms of a molecule can be performed and one can consider Eq. (1) as a sum over the molecules, with a molecular structure factor $[20] f(\mathbf{Q})$, the vectors $\mathbf{R}_{j}$ denoting positions of the mass centers of the molecules. The intramolecular disorder is treated here as statistically independent of the translational order of the mass centers of the molecules and leads to an effective DebyeWaller factor that is not considered here. The vectors $\mathbf{R}_{j}$ lie in the monolayer plane and the molecular structure factor $f(\mathbf{Q})$ is the only factor in the right-hand side of Eq. (1) that depends on the vertical component of the scattering vector Q. Integrating over this component, one arrives at a purely two-dimensional problem, with the vector $\mathbf{Q}$ in the layer plane. Since we do not compare the intensities of different reflections, the effective molecular factor (which includes also a Debye-Waller factor due to intramolecular disorder) is not essential for the considerations below and can be omitted.

The vector $\mathbf{R}_{j}$ can be represented as a sum $\mathbf{R}_{j}=\mathbf{r}_{j}+\mathbf{u}_{j}$ of the position $\mathbf{r}_{j}$ of a given molecule in the ideally periodic reference lattice and of its displacement $\mathbf{u}_{j}$ due to fluctuations of any nature. Accordingly, the scattering vector $\mathbf{Q}$ can be decomposed into a sum of two components, $\mathbf{Q}=\mathbf{Q}_{0}+\boldsymbol{\kappa}$, where $\mathbf{Q}_{0}$ is the reciprocal-lattice vector [so that $\mathbf{Q}_{0} \cdot \mathbf{r}_{j}$ $=2 \pi \times($ integer $)]$ and $\boldsymbol{\kappa}$ is a small deviation from it. The structure factor is given by the square of the amplitude (1) averaged over fluctuations in positions of the molecules, $s(\mathbf{Q})=\left\langle|A(\mathbf{Q})|^{2}\right\rangle$. Then, proceeding from sums to integrals, the structure factor near a given reflection $\mathbf{Q}_{0}$ can be represented as

$$
s(\mathbf{Q})=\int G_{\mathbf{Q}_{0}}(\mathbf{r}) \exp (i \boldsymbol{\kappa} \cdot \mathbf{r}) d^{2} r,
$$

where the integration is performed over the area of the coherently scattering domain and

$$
G_{\mathbf{Q}_{0}}(\mathbf{r})=\left\langle\exp \left\{i \mathbf{Q}_{0} \cdot[\mathbf{u}(\mathbf{r})-\mathbf{u}(0)]\right\}\right\rangle
$$

is the correlation function for the reflection $\mathbf{Q}_{0}$. For describing displacive disorder, the set of functions $G_{\mathbf{Q}_{0}}(\mathbf{r})$ is more convenient than van Hove's density-density correlation function $[21]\left\langle\rho\left(\mathbf{r}+\mathbf{r}^{\prime}\right) \rho\left(\mathbf{r}^{\prime}\right)\right\rangle$.

A two-dimensional powder consists of randomly oriented domains the contributions of which add incoherently. The average over domain orientations is equivalent to average over orientations of the scattering vector $\mathbf{Q}$ in a single domain, i.e., to integration of the structure factor over a circle $|\mathbf{Q}|=Q$, Fig. 2. The scattered intensity is concentrated close to the reciprocal lattice points and the structure factor $s(\mathbf{Q})$ is large only for $|\boldsymbol{\kappa}|$ small compared with the radius of the circle. Then the circle can be replaced by its tangent line and

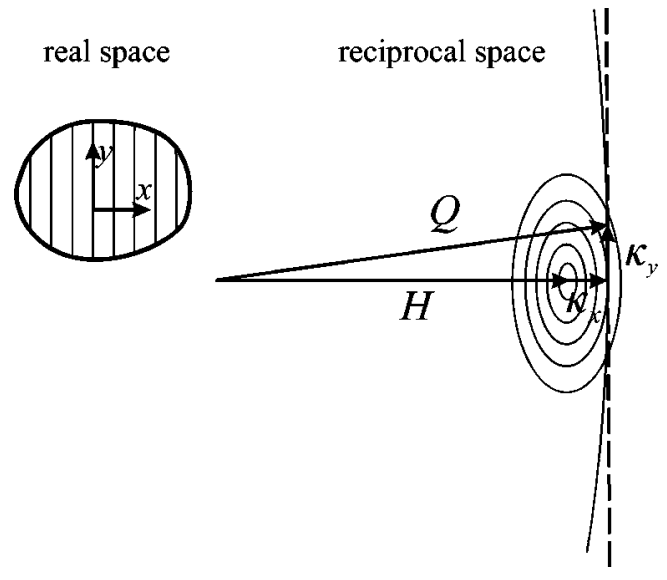

FIG. 2. Schematic diagram of the intensity averaging in a 2Dpowder diffraction experiment.

angular integration reduces to that over $d \kappa_{y} / Q$, where $\kappa_{y}$ is the component perpendicular to the reciprocal lattice vector $\mathbf{Q}_{0}$, see Fig. 2. In this integral, $Q$ in the denominator can be replaced by the constant $Q_{0}$. Integrating the structure factor (2) over $\kappa_{y}$, one obtains the delta function $\delta(y)$, where $y$ is the coordinate in the direction perpendicular to $\mathbf{Q}_{0}$. Then the powder-averaged structure factor $S_{\mathbf{Q}_{0}}\left(\boldsymbol{\kappa}_{x}\right)=\int s(\mathbf{Q}) d \boldsymbol{\kappa}_{y}$ simplifies to

$$
S_{\mathbf{Q}_{0}}(q)=\int G_{\mathbf{Q}_{0}}(x) \exp (i q x) d x
$$

where $q$ is the deviation from the peak position in the powder diffraction pattern, $q \equiv Q-Q_{0} \quad\left(q \approx \kappa_{x}\right.$ when the circle is replaced by the tangential line). Here $x$ is the coordinate in the direction of $\mathbf{Q}_{0}$, and the correlation function $G_{\mathbf{Q}_{0}}(x)$ $\equiv G_{\mathbf{Q}_{0}}(x, y=0)=G_{\mathbf{Q}_{0}}\left(x \mathbf{Q}_{0} / Q_{0}\right)$ describes positional correlations between two points separated by the distance $x$ in the direction along the reciprocal-lattice vector $\mathbf{Q}_{0}$. This simple representation (4) of the structure factor as a onedimensional Fourier integral of the correlation function allows us to determine the correlation function directly from the measured profile of the diffraction peak by applying the inverse Fourier transform.

Another derivation of Eq. (4), which also shows the way for a systematic improvement of this approximation, can be made by first integrating Eq. (2) over the orientations of $\mathbf{Q}$ to obtain

$$
\begin{aligned}
S_{\mathbf{Q}_{0}}(q)= & \iint d x d y G_{\mathbf{Q}_{0}}(x, y) \\
& \times J_{0}\left(\left(Q_{0}+q\right) \sqrt{x^{2}+y^{2}}\right) \exp \left(-i Q_{0} x\right),
\end{aligned}
$$

where $J_{0}(z)$ is the Bessel function. The last two multipliers in the right-hand side of Eq. (5) are rapidly oscillating functions, since $Q r \gg 1$. The main contribution to the integral comes from regions where their phases compensate each other. Using the asymptotics of the Bessel function at large arguments and expanding $r=\sqrt{x^{2}+y^{2}}$ for $y \ll x$, one arrives at Eq. (4). Further improvement of this approximation can be made within the framework of the stationary phase method. A similar three-dimensional problem of $\mathrm{x}$-ray scattering in a 

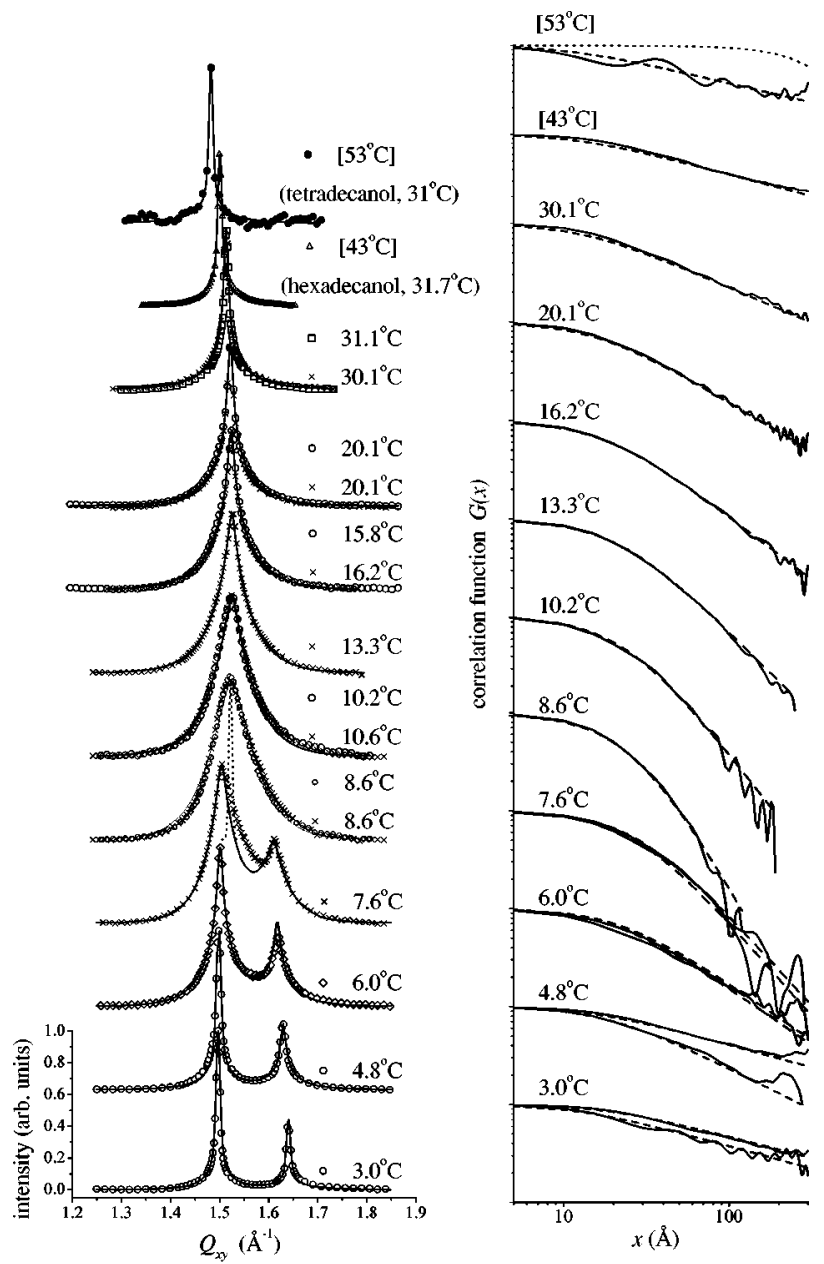

FIG. 3. X-ray diffraction peaks (left column) and correlation functions (right column) for octadecanol monolayers at a surface pressure of $18 \mathrm{mN} / \mathrm{m}$. Different symbols correspond to data from monolayers prepared in different ways (see text). The full lines are fits of the structure factor (7) convolved with the Gaussian approximation of the resolution function. The dotted line shows the resolution function. The widths of all peaks exceed the resolution limit. In the right column, full lines present Fourier transforms of the measured peaks, divided by the Fourier transform of the resolution function (shown by the dotted line). Dashed lines show the correlation functions given by Eq. (6) with the same values of $\eta$ and $\xi$ as the fits to the peaks in the left column.

powder sample of a smectic liquid crystal was considered in Ref. [22]. Using Eq. (5) instead of Eq. (4) leads mainly to an asymmetry of the peak profile. The present study did not reveal a noticeable asymmetry in the observed diffraction peaks of Langmuir monolayers and we restrict ourselves to the approximation (4).

\section{B. Positional correlations in octadecanol monolayers}

Figure 3 (left column) shows the measured diffracted intensities corrected for Lorentz factor, effective area, and polarization, with a linear background subtracted, and integrated over the vertical component $Q_{z}$ of the scattering vector. The temperature variation of the diffraction pattern is in agreement with a $L S-S$ phase transition at $8{ }^{\circ} \mathrm{C}[8-11]$. Further analysis of the data shows (Sec. III C) that the transition is of first order. Below this temperature (phase $S$ ), one observes two diffraction peaks characteristic of the centered rectangular unit cell [23]. Above $8{ }^{\circ} \mathrm{C}$ (phase $L S$ ) there is only one peak due to a hexagonal arrangement of the molecules. This peak becomes sharper with increasing temperatures. The present experiment did not reproduce our previous observation [12], performed during continuous heating of the monolayer, of two overlapping broad peaks in the temperature range $8-10^{\circ} \mathrm{C}$, similar to the diffraction pattern of the $L S_{I}$ phase in fatty acids [25]. These probably point to a nonequilibrium nature of this diffraction pattern. Strong kinetic effects, with relaxation from centered rectangular to hexagonal structure within tens of minutes, have been reported for this part of the phase diagram [26].

Figure 3 comprises peaks measured on monolayers prepared in several different ways. One of the monolayers (the data points denoted by circles) was compressed to $18 \mathrm{mN} / \mathrm{m}$ at $3{ }^{\circ} \mathrm{C}$, then expanded to zero surface pressure, heated and compressed again for new measurements. This monolayer was compressed and expanded 11 times with subsequent heating after each expansion in the temperature interval from 3 to $20^{\circ} \mathrm{C}$. Another monolayer (crosses) was compressed first at $7.6{ }^{\circ} \mathrm{C}$ and then heated to $31{ }^{\circ} \mathrm{C}$ keeping the surface pressure constant. A third monolayer (squares) was compressed for the first time at $31{ }^{\circ} \mathrm{C}$. The diffraction peaks obtained from different monolayers at similar temperatures coincide, thus proving that the measurements were performed in equilibrium states of the monolayer and that the result does not depend on its previous processing.

Additional measurements were performed on shorter chain alcohols, hexadecanol and tetradecanol, with the aim of expanding the effective temperature range. Taking into account that shortening the chain by two methylene groups is equivalent to increasing the temperature by $10-12^{\circ} \mathrm{C}[27]$, the state of the hexadecanol monolayer at $31.7^{\circ} \mathrm{C}$ and that of the tetradecanol monolayer at $31^{\circ} \mathrm{C}$ approximately correspond to the states of the octadecanol monolayer at 43 and $53{ }^{\circ} \mathrm{C}$, respectively. At $31^{\circ} \mathrm{C}$ tetradecanol exhibits a noticeable solubility. In order to keep the pressure constant, the monolayer area was continuously decreasing during the measurement. This restricted the measurement time and, as a result, the tetradecanol data in Fig. 3 are more scattered.

Figure 4 shows some peaks and the processing of the diffraction data in greater detail. The resolution function is shown in the left column by dashed lines. The widths of all peaks exceed the resolution limit. The measured intensity $\mathcal{I}_{\mathbf{Q}_{0}}(q)$ is the convolution of the structure factor $S_{\mathbf{Q}_{0}}(q)$ with the resolution function $R(q), \quad \mathcal{I}_{\mathbf{Q}_{0}}(q)=S_{\mathbf{Q}_{0}}(q) \otimes R(q)$. Then, Fourier transforming this equation, one obtains the correlation function as a ratio $G_{\mathbf{Q}_{0}}(x)=\widetilde{\mathcal{I}}_{\mathbf{Q}_{0}}(x) / \widetilde{R}(x)$, where the tilde denotes Fourier transforms of the corresponding functions. The right column of Fig. 4 shows the real-space functions. The Fourier transform $\widetilde{R}(x)$ of the resolution function is shown by dotted lines. The Fourier transform $\widetilde{\mathcal{I}}_{\mathbf{Q}_{0}}(x)$ of the peak is shown by thin solid lines. The resolutioncorrected correlation functions, i.e., the ratios $G_{\mathbf{Q}_{0}}(x)$ $=\widetilde{\mathcal{I}}_{\mathbf{Q}_{0}}(x) / \widetilde{R}(x)$, are shown by thick solid lines.

The full width at half maximum $\mathcal{W}$ of the resolution function in the horizontal plane gives rise to a distance $x_{m}$ $=\pi / \mathcal{W} \approx 380 \AA$ available in the experiment (the commonly 

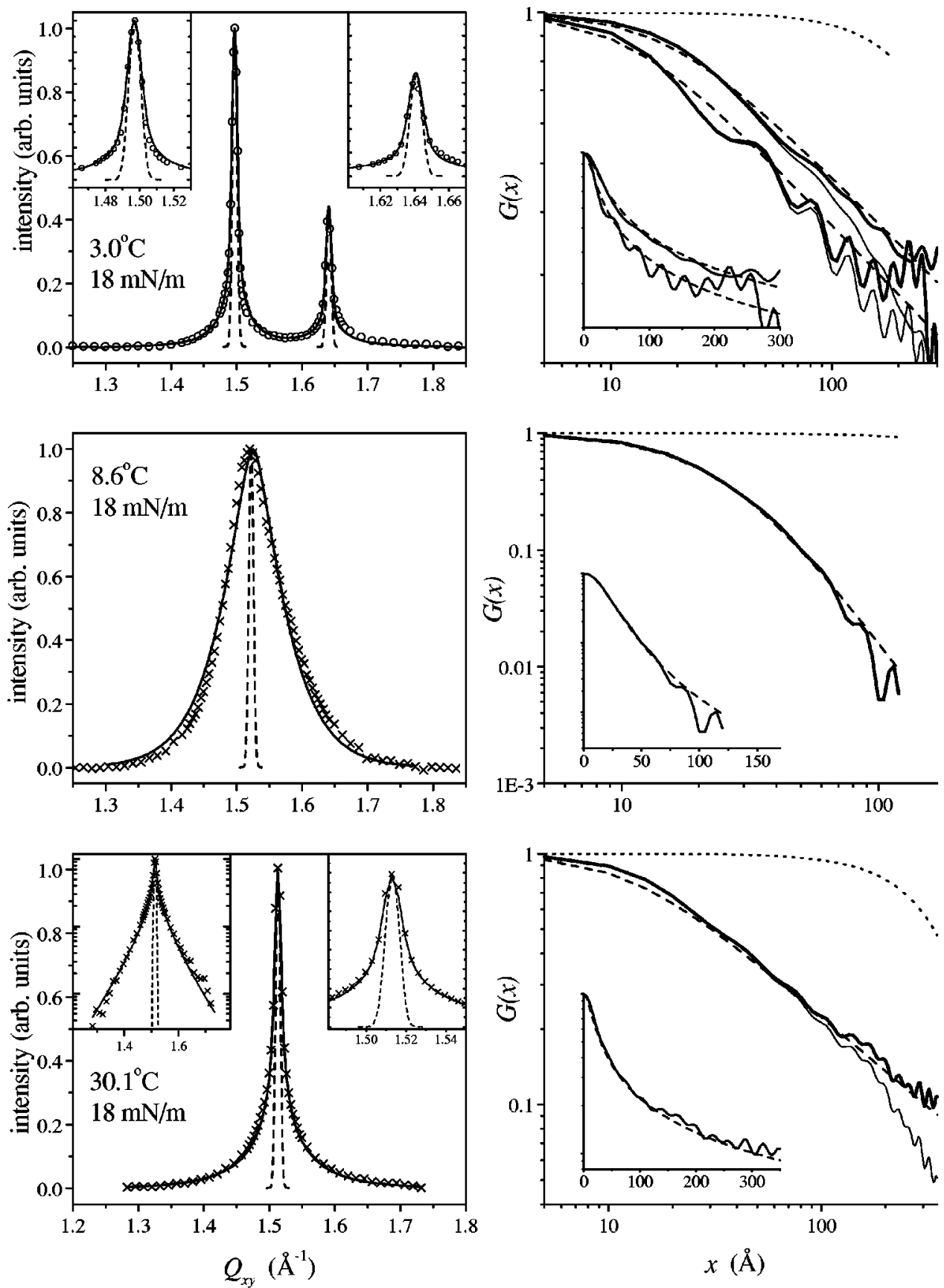

FIG. 4. Some peaks from Fig. 3 are shown in more detail. The inserts in the left column show the same peaks in different scales. The inserts in the right column show the correlation functions on a single-logarithmic scale.

used value $2 \pi / \mathcal{W}$ describes the interval $-x_{m}<x<x_{m}$ ). In principle, one could obtain the correlation function even for distances exceeding $x_{m}$, provided the resolution function is accurately specified, the scatter of the measured data points and the interval $\Delta q$ between the measured points are small enough. In the present study a rather large step $\Delta q$ $=0.0125 \AA^{-1}$ between the data points in the wings of the peaks (the central parts of the peaks were measured with a four times smaller step) limits the range of $x$ to $\pi / \Delta q$ $\approx 250 \AA$ A. At larger $x$, the correlation functions obtained by direct Fourier transformation of the experimental data (without any smoothing) show strong oscillations. The problem could be avoided by an appropriate interpolation of the data. This, however, was not necessary since we also fitted the peaks directly, as is described below. The fits show that the behavior of the correlation function is not changed on somewhat larger distances.

The diffraction peak of the $L S$ phase (with a hexagonal unit cell) is due to six equivalent reflections that overlap after powder average. The correlation function $G_{\mathbf{Q}_{0}}(x)$ represents correlations along each of them. In the $S$ phase the two peaks in the powder diffraction pattern are due to nonequivalent diffraction vectors of the rectangular unit cell. These peaks were processed independently giving rise to different correlation functions in the directions of the corresponding diffraction vectors.

One can see from the right columns of Figs. 3 and 4 that the correlation functions are close to straight lines in the 
double-logarithmic scale both above and below the transition, thus demonstrating the algebraic decay of positional correlations. When replotted in a single-logarithmic scale (insets in Fig. 4), these lines are far from being straight. The only exception is the correlation function at $8.6^{\circ} \mathrm{C}$, close to the transition, which can be equally well fitted by a power law decay or by an exponential decay. There is no evidence for a characteristic length that would restrict the algebraic decay from the side of large $x$.

From the small- $x$ side, the power law is seen to be restricted to a temperature-dependent length $L$ of the order of $10 \AA$. Hence the correlation functions may be conveniently interpolated by the function

$$
G_{\mathbf{Q}_{0}}(x)=\left[1+\left(\frac{x}{L}\right)^{2}\right]^{-\eta / 2},
$$

which contains a characteristic size $L$ and possesses an algebraic decay $G_{\mathbf{Q}_{0}}(x) \propto 1 / x^{\eta}$ at $x \gg L$. The functional form of Eq. (6) is just a convenient interpolation and there is no physical meaning behind it; it merely helps to characterize the correlation functions by the appropriate parameters, $L$ and $\eta$.

An advantage of Eq. (6) is the possibility of performing the Fourier transformation analytically:

$$
S_{\mathbf{Q}_{0}}(q)=|q|^{(\eta-1) / 2} K_{(\eta-1) / 2}(L|q|),
$$

where $K_{m}(z)$ is a modified Bessel function. Equation (7) provides a convenient interpolation formula describing (after convolution with the resolution function) the shape of the diffraction peak. In the limit $q \rightarrow 0$, Eq. (7) gives rise to a singularity $S_{\mathbf{Q}_{0}}(q) \propto q^{-1+\eta}$ when $\eta<1$ or to a cusp when $\eta>1$. The structure factor (7) may be used to fit the peaks when the scatter of the experimental data prevents direct application of the Fourier transformation to obtain the correlation function. The curves in the left column of Figs. 3 and 4 are obtained by numerically convolving Eq. (7) with the Gaussian approximation for the resolution function. The values of $\eta$ and $L$ thus obtained are used to calculate the correlation functions by Eq. (6). The results are shown in the right columns of Figs. 3 and 4 by dashed lines. They are in a good agreement with the correlation functions obtained directly by Fourier transformation of the peak profiles.

The fit of Eq. (7) to the data requires proper subtraction of the background scattering from the water. We measured the intensity in a range of scattering vectors sufficiently wide to reach the background level on both sides of the peaks. Prior to integration over $Q_{z}$, the background was subtracted by interpolation of the intensity observed far from the peak in $\left(Q_{x y}, Q_{z}\right)$ space, assuming that the background depends linearly on the in-plane $\left(Q_{x y}\right)$ and the vertical $\left(Q_{z}\right)$ components of the wave vector transfer. A linear variation of the background in the relevant range of scattering angles is only an approximation. Measurement of the scattering from the pure water surface in the same range of the scattering angles also does not completely resolve this problem since the influence of the monolayer on the background scattering is not precisely known. In practice, the parameters $\eta$ and (to a lesser extent) $L$ are not sensitive to small variations of the background level because these variations leave the central in-

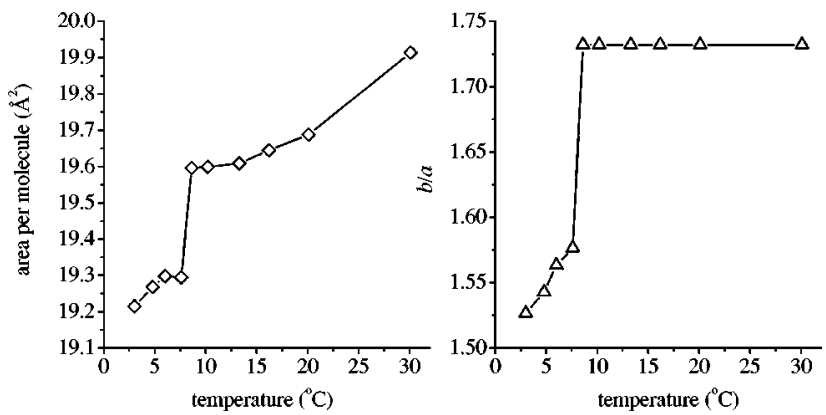

FIG. 5. Temperature dependencies of the area per molecule $a b / 2$ and the ratio $b / a$ of the parameters $a, b$ of the rectangular unit cell derived from the positions of diffraction peaks.

tense part of the peak almost unchanged and hence do not alter the large- $x$ part of the correlation function. An error in the background subtraction can be essential at the wings of the diffraction peak and may lead to incorrect description of the short-range features of the resulting correlation function. We assume that the background is 'properly' subtracted when the wings of the peak plotted on a logarithmic scale (see the insert on the bottom of Fig. 4) are close to straight lines, which corresponds to the (large- $q$ ) asymptotically exponential decay of the modified Bessel function in Eq. (7) and we do not introduce an additional small-length parameter.

\section{Temperature dependencies of structure and correlation parameters}

Figure 5 presents the temperature dependencies of the area per molecule $a b / 2$ and the ratio $b / a$ of the sides of the rectangular unit cell determined from positions of the diffraction peaks. At the first-order transition $L S-S$, the area per molecule decreases by approximately $1.5 \%$ while the unit cell distorts by about $9 \%$ in $b / a$ with respect to a hexagonal cell $(b / a=\sqrt{3})$.

Figure 6 shows the temperature dependencies of the exponent $\eta$ and the length $L$ as determined from the fits of Eq. (7) to the peak profiles. When, in the $S$ phase, two peaks are observed and processed separately, two values result and their mean is presented in Fig. 6. Both parameters exhibit
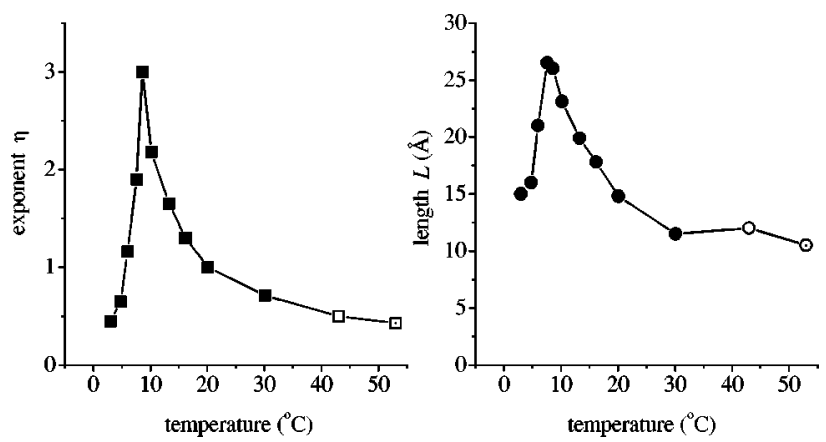

FIG. 6. Temperature dependencies of the exponent $\eta$ and the length $L$ derived from fits of Eq. (7) to the peaks in Fig. 3. Full symbols correspond to the octadecanol monolayers. Open symbols correspond to the hexadecanol and tetradecanol monolayers at $31^{\circ} \mathrm{C}$, with the temperature axes shifted by $11^{\circ} \mathrm{C}$ per two methylene groups. 

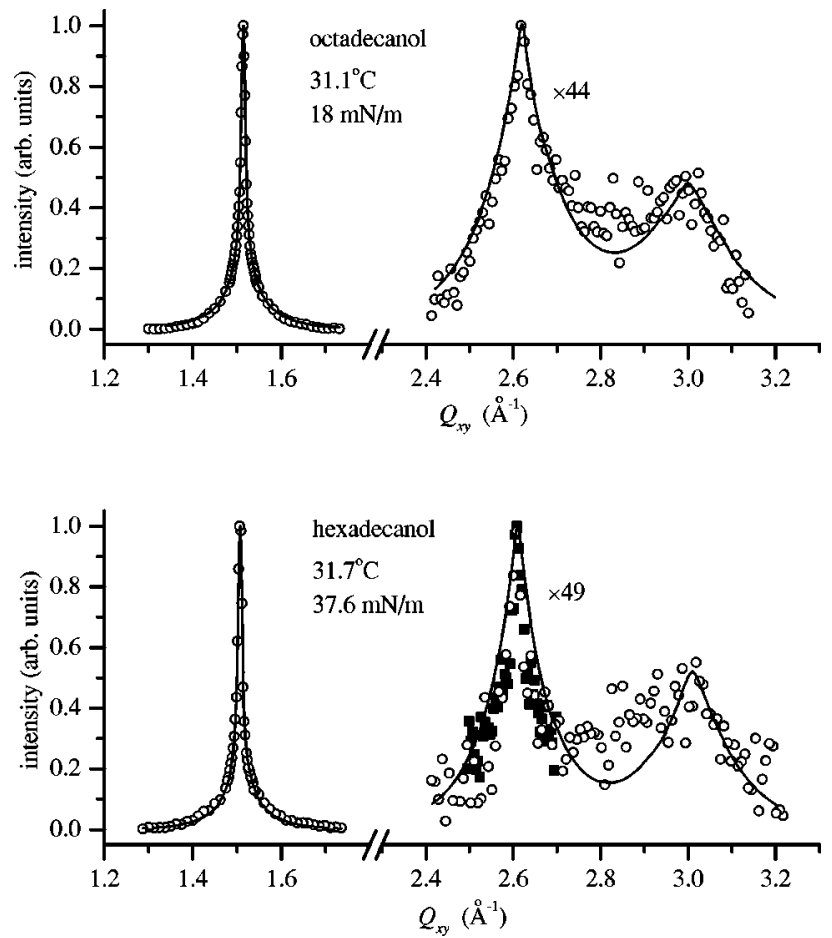

FIG. 7. Higher-order peaks from monolayers of octadecanol and hexadecanol and their simulation using Eq. (7). Different symbols denote independent measurements. The positions of the higherorder peaks $Q_{i}$ were determined from the positions of the first-order peaks, the exponent $\eta$ was scaled as $\eta_{i} \propto Q_{i}^{2}$, and the length $L$ was the same as for the first-order peaks.

maxima at the $L S-S$ transition temperature $\left(8^{\circ} \mathrm{C}\right)$. The length $L$ is comparable with the thickness of the monolayer $(\approx 22.5 \AA)$ and the simplest interpretation of this parameter could be as the crossover from two-dimensional to threedimensional behavior, since algebraic decay of positional order occurs in thin crystalline films at distances exceeding the film thickness [28]. However, the systematic temperature variation of $L$ suggests another origin of this parameter. Note that $L$ varies from approximately 3 to 5 lattice spacings ( $a$ $=4.8 \AA$ ). The origin of the algebraic decay and the temperature dependencies of the parameters $\eta$ and $L$ are discussed in Sec. IV.

\section{Higher-order peaks}

For scattering due to lattice distortions, regardless of their nature, one expects that the exponent of algebraic decay is proportional to the square of the diffraction vector, $\eta_{i}$ $\propto Q_{i}^{2}$. This dependence was verified by measuring higherorder diffraction peaks of the hexagonal phase $L S$, Fig. 7. Using the common notation of the peaks, which refers to the centered rectangular unit cell of the phase $S$, the observed higher-order peaks can be labeled as (20) and (13). The peaks are very weak and the scatter of the data points is large, which prevents direct Fourier transformation of the measured peaks in the same way as was done with the loworder peaks. Instead, we used the values of the parameters determined from the first-order peaks to simulate the higherorder peaks. Specifically, the positions of the observed higher-order peaks were determined from the scattering vec-
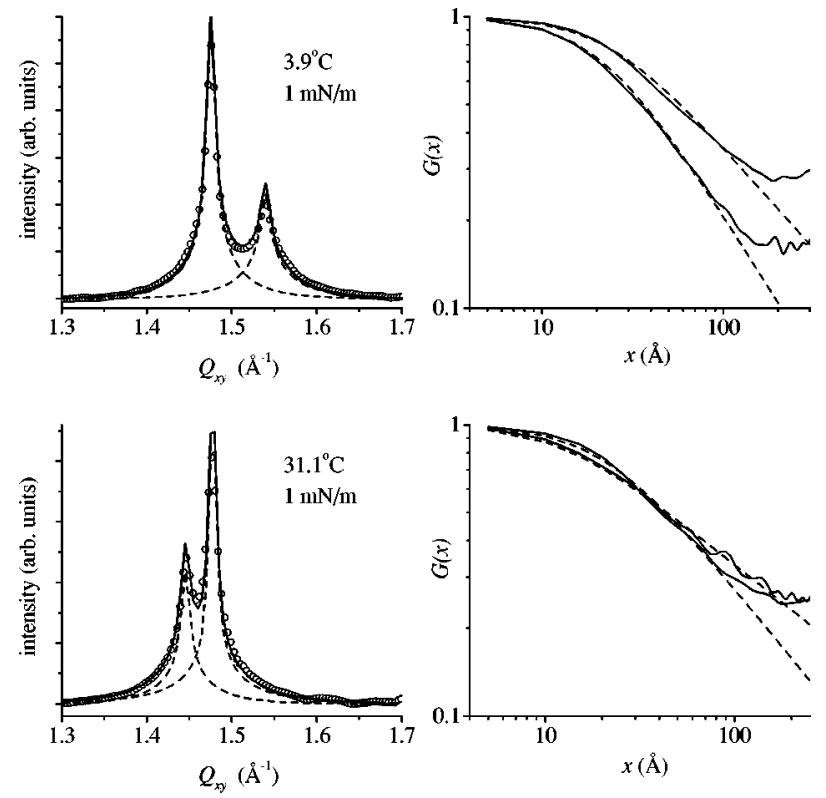

FIG. 8. Peak profiles and correlation functions for the monolayer in the tilted state at the surface pressure of $1 \mathrm{mN} / \mathrm{m}$. The line-types are as in Fig. 3.

tor $Q_{1}$ of the first-order peak as $Q_{2}=\sqrt{3} Q_{1}$ and $Q_{3}=2 Q_{1}$. The exponents $\eta$ were taken as $\eta_{i}=\eta_{1}\left(Q_{i} / Q_{1}\right)^{2}$, where $\eta_{1}$ is the value determined from the first-order peak, and the length $L$ was the same as obtained from the first-order peak. The good agreement between the data and the structure factors calculated by Eq. (6) with these values of the parameters confirms that the broadening is caused by distortions of the lattice.

\section{E. Positional order in tilted phases}

Figure 8 shows diffraction peaks measured at a low surface pressure of $1 \mathrm{mN} / \mathrm{m}$, in the tilted state of the octadecanol monolayer, at both high and low temperatures. Our aim was to test if the same analysis of the peak profiles can be applied to the tilted phases but we did not attempt to study the tilted phases systematically. Both peaks are out of the water plane, the weaker peak, characteristically of the NNN-tilted phase $[6,29]$, having a twice as large value of the vertical component of the scattering vector $Q_{z}$. The tilt angles determined from the Bragg rod profiles [6] are $17.7^{\circ}$ and $18.7^{\circ}$ at 3.9 and $31.1{ }^{\circ} \mathrm{C}$, respectively.

The in-plane shape of the peaks can be adequately described by Eq. (7) and the correlation functions exhibit algebraic decay, Fig. 8. At a low temperature of $3.9^{\circ} \mathrm{C}$, the difference between the correlations along different diffraction vectors is more pronounced. The values of the exponent $\eta$, 0.42 and 0.33 at 3.9 and $31.1^{\circ} \mathrm{C}$, respectively, are somewhat smaller than those in the untilted state, cf. Fig. 6. On the other hand, the values of the length $L, 22$ and $16 \AA$, respectively, are larger than in the untilted state (the values of the parameters presented here are, as above, the mean of the values given by two peaks).

\section{F. Compressibility of octadecanol monolayers}

We measured the compressibility of the monolayer at several temperatures, with the aim of applying it in the calcula- 

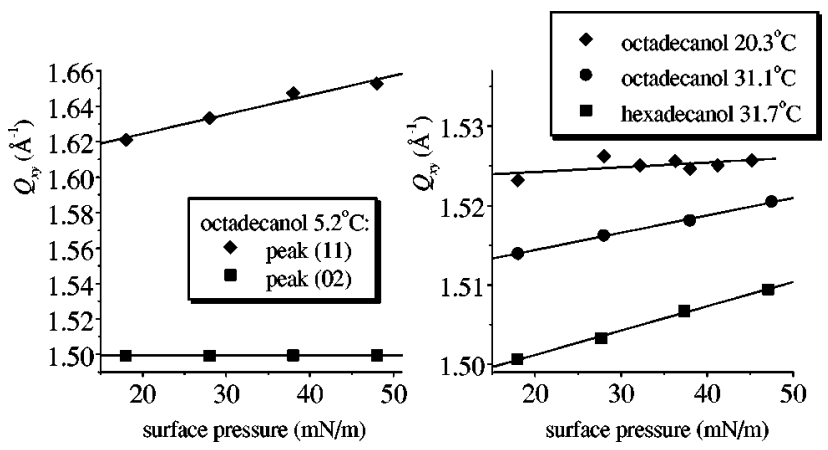

FIG. 9. Pressure dependence of the peak positions in monolayers of octadecanol and hexadecanol.

tion of the exponent $\eta$ in the next section. The compressibility $\chi$ is the relative change of the area $A$ in response to the change of surface pressure $\Pi$, i.e., $\chi=-A^{-1}(d A / d \Pi)$. The reciprocal quantity is the compression modulus $K=1 / \chi$ $=-A(d \Pi / d A)$. In the hexagonal phase it can be directly expressed via the change in position of the diffraction peak on applied surface pressure, $K=-\frac{1}{2} Q_{0}\left(d \Pi / d Q_{0}\right)$. Only isotropic compression can be applied to a powder sample; hence, the shear elastic constant cannot be determined. In phases with a rectangular unit cell, the linear compressibilities along each diffraction vector $\mathbf{Q}_{h k}$ can be determined as $\chi_{h k}=-Q_{h k}^{-1}\left(d Q_{h k} / d \Pi\right)$ [30].

Figure 9 presents the pressure dependencies of the positions of the diffraction peaks at different temperatures. The two peaks of the phase $S$ (Fig. 9, left), show quite different responses to the applied pressure: the position of the nondegenerate (02) peak remains unchanged within the accuracy of the measurements, while the compressibility in the direction of the degenerate (11) peak is $\chi_{11}=0.67 \mathrm{~m} / \mathrm{N}$. The area compressibility is then $\chi=\chi_{11} / \cos ^{2}\left(\gamma^{*} / 2\right) \simeq 0.85 \mathrm{~m} / \mathrm{N}$ and $K \simeq 1.18 \mathrm{~N} / \mathrm{m}$. Here, $\gamma^{*}$ is the angle between the $\mathbf{Q}_{11}$ and $\mathbf{Q}_{1 \overline{1}}$ reciprocal lattice vectors (cf. [31]). A similar anisotropy and close values of the compressibility were found in the phase $S$ of behenic acid [30]. The compressibility of the phase $L S$ is much lower (Fig. 9, right). (This phase was not studied in Ref. [30].) From linear fits we found values $\chi$ $=0.41,0.29$, and $0.08 \mathrm{~m} / \mathrm{N}$ for hexadecanol at $31.7^{\circ} \mathrm{C}$ and octadecanol at 31.1 and $20.3^{\circ} \mathrm{C}$, respectively. The last value is smaller than the compressibility of the crystalline phase $C S$ of behenic acid $(\chi \approx 0.15 \mathrm{~m} / \mathrm{N})$ [30]. From the values given above, the compression modulus $K=1 / \chi$ takes on the values $2.4,3.45$, and $12.5 \mathrm{~N} / \mathrm{m}$, respectively.

Attempting to determine the compressibility of octadecanol at $11.5^{\circ} \mathrm{C}$, close to the transition $L S-S$, we occasionally observed [32] this phase transition on compression, rather than by cooling, Fig. 10. The transition pressure is approximately $40 \mathrm{mN} / \mathrm{m}$. Although the observation of such a transition is not surprising ( $L S-S$ phase transition lines inclined to higher temperatures on increasing surface pressure have been reported in many isotherm and Brewster angle microscopy studies), this is, to the best of our knowledge, the first diffraction observation of the backbone-ordering transition at a constant temperature. In the related bulk system, rotator phases of bulk alkanes, the transition temperatures also increase under the action of the high pressure gas [33]. Thus, the $L S-S$ phase transition line in the phase diagram of

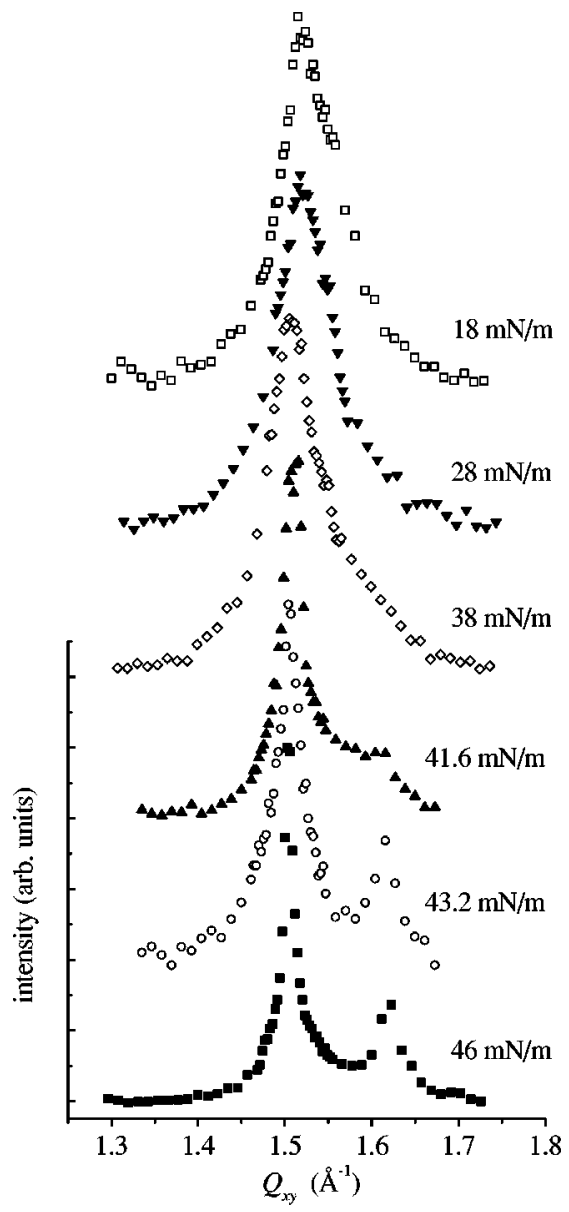

FIG. 10. Diffraction peaks along the $11.5^{\circ} \mathrm{C}$ isotherm. A phase transition is seen between 38 and $41.6 \mathrm{mN} / \mathrm{m}$.

octadecanol $[8-11]$ is inclined towards higher temperatures on increasing the surface pressure. The broad diffraction peaks in the $L S$ phase at this temperature indicate that the monolayer is in a disordered state close to the transition and the compressibility cannot be determined from the positions of the maxima. Apparently the peak position $Q_{0}$ decreases on compression from 18 to $38 \mathrm{mN} / \mathrm{m}$, which would correspond to a thermodynamically impossible negative compressibility.

\section{G. Comparison of monolayer and bulk systems}

The results of the present study can be compared with the detailed x-ray diffraction studies of the closely related bulk system, rotator phases of $n$-alkanes, by Sirota and co-workers (see Ref. [34] and papers cited therein). The latter system is a three-dimensional crystal consisting of weakly coupled layers, with the hydrocarbon chains aligned parallel to each other either normally to the layer or tilted. The coupling between layers gives rise to the diffraction pattern of a threedimensional crystal, which consists of two components [19]: a $\delta$-shaped Bragg peak reflecting the long-range translational order and the diffuse scattering around it due to disorder in the positions of the molecules. The Bragg peaks appear weak because of the large mean square displacements $\left\langle u^{2}\right\rangle$ and are experimentally not distinguished from the strong diffuse scattering, pointing to strong positional disorder in the rotator phases of $n$-alkanes. In a two-dimensional solid, by con- 
trast, the mean square displacements $\left\langle u^{2}\right\rangle$ diverge [1], true Bragg peaks are absent, and the power-law decay of the structure factor reflects quasi-long-range positional order.

The two systems exhibit very similar microscopic structures and, what is more important in the context of the present discussion, similar temperature dependencies of the peak shapes. In the rotator phases of mixtures of $n$-alkanes [34,35], broadening of the diffraction peak of the hexagonal phase $R_{\mathrm{II}}$ on cooling, similar to the observations presented above, was found. Mixing alkanes of different chain lengths weakens the coupling between the layers and makes the behavior of the bulk system more similar to that of a monolayer. The peak broadening was attributed $[34,35]$ to scattering from domains of the backbone-ordered phase $R_{\mathrm{I}}$ with the orthorhombic unit cell. In the monolayer system, similar disorder manifests itself in an increase of the exponent $\eta$, which points to strains in the matrix around the fluctuation as a source of scattering (see discussion in the next section). The characteristic size of the fluctuation, given by the length $L$, remains small (several lattice spacings) even close to the transition.

Another similarity between two the systems can be found in the variation of the peak profiles close to the transition. In the mixtures of $n$-alkanes in the hexagonal phase $R_{\text {II }}$ close to the transition to the orthorhombic phase $R_{\mathrm{I}}$, the Bragg component of the peak apparently disappears, which probably indicates a transition from the hexagonal crystal to a stacked hexatic [35]. Similarly, the observations on octadecanol monolayers at $8.6^{\circ} \mathrm{C}$ presented in Fig. 4 may mean a transition from the power-law decay to the exponential decay of positional correlations.

Any x-ray diffraction study probes positional order on a finite length scale limited by the experimental resolution. Attempts to extend the results to infinity remain ambiguous. Additional, in particular thermodynamical, arguments have to be taken into account to characterize the long-range order in the system. Similarities between structures of phases in two systems gave rise to two diverse interpretations of these systems [34,36]. Sirota [34] suggested that all phases of Langmuir monolayers are solids and the finite widths of the diffraction peaks are a result of low resolution, rather than finite size of the coherently scattering domains. By contrast, Peterson and Kenn [36] suggested, based on the similarity of the monolayer phases to the liquid crystalline ones and on thermodynamical arguments, that the phases of both systems are hexatics. In our opinion, neither of these suggestions have yet been proven. The present study reveals algebraic decay of positional correlations which proves the solidlike order in the phases $L S$ and $S$ of the octadecanol monolayers on the length scale available in present diffraction experiment. This length scale is rather limited $(<800 \AA)$ and does not allow conclusions on the positional order at longer distances. The accessible length scale can be significantly increased (by a factor $\geqslant 50$, cf. [7]) with the use of the new generation of synchrotron sources. Designating the phase $L S$ as a solid seems in disagreement with its small viscosity, which decreases close to the transition $L S-S$ and which gave the name ("superliquid") to this phase [37]. Designating the phase $S$ as a solid leads to a question as to the nature of the $S-C S$ transition, which was treated [3] as a crystallization of the mesophase. Therefore, in our opinion, the description of the phases $S$ and $L S$ as either solids or fluids remains ambiguous and requires further studies, in particular, higherresolution diffraction experiments.

\section{INTERPRETATION OF POSITIONAL CORRELATIONS}

The aim of this section is to consider possible sources of the observed algebraic decay with anomalously large exponents $\eta$. In addition to the most general source of algebraic decay, long-wavelength thermal phonons in a twodimensional solid [1], we consider thermal fluctuations specific to the observed structural phase transition $L S-S$. These fluctuations, with the structure of the backbone-ordered phase, distort the unit cell and cause elastic strains in the surrounding matrix, Fig. 1. The algebraic decay of the correlation function due to these strains is a consequence of the dimensionality of the system.

The correlation function (3) can be written in the harmonic approximation as $G_{\mathbf{Q}_{0}}(\mathbf{r})=\exp [-U(\mathbf{r})]$, where

$$
\begin{aligned}
U(\mathbf{r}) & =\frac{1}{2}\left\langle\left[\mathbf{Q}_{0} \cdot(\mathbf{u}(\mathbf{r})-\mathbf{u}(0))\right]^{2}\right\rangle \\
& =\sum_{\mathbf{k}}(1-\cos \mathbf{k} \cdot \mathbf{r})\left\langle\left|\mathbf{Q}_{0} \cdot \mathbf{u}_{\mathbf{k}}\right|^{2}\right\rangle .
\end{aligned}
$$

Proceeding from the sum in Eq. (8) to an integral and approximating the angular integral over the orientations of $\mathbf{k}$ by the product of the angular integrals of each of the factors one gets

$$
U(r)=\frac{\Omega}{2 \pi} \int\left[1-J_{0}(k r)\right] \overline{\left\langle\left|\mathbf{Q}_{0} \cdot \mathbf{u}_{\mathbf{k}}\right|^{2}\right\rangle} k d k,
$$

where $\Omega$ is the area of the system or its relevant domain and the bar denotes angular average. The error introduced by this commonly used approximation $[1,38,39]$ was considered in Ref. [40] and is small at both small and large $r$. Consider first the well-known source of displacements, long-wavelength thermal phonons. Their spectrum is governed by the elastic free energy $F_{\mathrm{el}}=\frac{1}{2} \int c_{i j l m} u_{i j} u_{l m} d^{2} r$, where $c_{i j l m}$ is the elastic tensor and $u_{i j}=\left(\partial u_{i} / \partial x_{j}+\partial u_{j} / \partial x_{i}\right) / 2$ is the strain tensor. Proceeding to the Fourier transform, one obtains

$$
F_{\mathrm{el}}=\frac{\Omega}{2} \sum_{\mathbf{k}} k^{2} C_{i l} u_{i \mathbf{k}} u_{l \mathbf{k}}^{*}
$$

where $C_{i l}(\mathbf{n})=c_{i j l m} n_{j} n_{m}$ and $\mathbf{n}=\mathbf{k} / k$ is the unit vector in direction of the wave vector. Then one readily finds that $\left\langle u_{i \mathbf{k}} u_{l \mathbf{k}}^{*}\right\rangle=k_{\mathrm{B}} T C_{i l}^{-1} / \Omega k^{2}$, where $C_{i l}^{-1}$ denote components of the tensor inverse to $C_{i l}$. In an elastically isotropic solid, one has $C_{i l}=\mu \delta_{i l}+(\lambda+\mu) n_{i} n_{l}$, where $\lambda$ and $\mu$ are Lamé coefficients, and the inverse tensor is easily calculated as $C_{i l}^{-1}$ $=\mu^{-1}\left[\delta_{i l}-n_{i} n_{l}(\lambda+\mu) /(\lambda+2 \mu)\right]$. Averaging $\left\langle\left|\mathbf{Q}_{0} \cdot \mathbf{u}_{\mathbf{k}}\right|^{2}\right\rangle$ $=k_{\mathrm{B}} T\left(\mathbf{Q}_{0} \cdot \hat{\mathbf{C}}^{-1} \cdot \mathbf{Q}_{0}\right) / \Omega k^{2}$ over the orientations of the vector $\mathbf{n}$, one obtains

$$
U(r)=\eta \int_{0}^{k_{\max }} \frac{d k}{k}\left[1-J_{0}(k r)\right] .
$$


It is convenient to proceed from the Lamé coefficients $\lambda$ and $\mu$ to the compression modulus $K=\lambda+\mu$ and the Poisson ratio $\sigma=\lambda /(\lambda+\mu)$ and express $\eta$ as

$$
\eta=\frac{k_{\mathrm{B}} T}{4 \pi K} \frac{(1+\sigma)(3-\sigma)}{2(1-\sigma)} Q_{0}^{2} .
$$

The integral (11) converges at $k \rightarrow 0$ while the largest wave vector $k_{\max }$ in a solid film is given by the film thickness. For $k>k_{\max }$ the film behaves as a three-dimensional solid [28].

Doing the integral (11) by parts, one finds $U(r)$ $\approx \eta \ln \left(k_{\max } r\right)$ and hence $G_{\mathbf{Q}_{0}}(r) \propto r^{-\eta}$. The theory of dislocation-mediated melting predicts [2] a phase transition from the hexagonal 2D solid to a hexatic at a temperature $T$ given by $k_{\mathrm{B}} T Q_{1}^{2} / 4 \pi K=(1-\sigma) / 6$. Here $Q_{1}$ is the first-order reciprocal lattice vector of the hexagonal lattice related to the lattice spacing $a$ by $Q_{1}=4 \pi / \sqrt{3} a$. Then the first-order diffraction peak at the melting temperature has the exponent $\eta_{c}=(1+\sigma)(3-\sigma) / 12$, which reaches the maximum value of $1 / 3$ when the Poisson ratio takes its maximum value $\sigma$ $=1$.

Zakri et al. [7] found that the monolayer of decanol on the surface of water in contact with a drop of liquid decanol possess 2D-crystalline order (on the scale of at least thousands of $\AA$ ) with the exponent of the algebraic decay increasing at the melting transition of the crystal. The maximum value that they found was $\eta=0.57$ which exceeds the limit given by the theory of dislocation-mediated melting. Note that this value is close to the smallest values found in the present study far from the $L S-S$ transition, Fig. 6. It was suggested [7] that the dislocation-mediated melting scenario is irrelevant because of a very high energy of nucleating of dislocation pairs. Then the exponent (12) can be large, provided the Poisson ratio $\sigma$ is close to 1 . The compression modulus $K$ can be measured independently and $\sigma$ remains the only unknown. Conversely, it can be determined from the observed value of $\eta$. The ratio of the shear modulus $\mu$ $=[(1-\sigma) /(1+\sigma)] K$ to the compression modulus $K$ was found to be fairly small, $\mu / K \approx 0.04$ close to the melting transition [7].

Applying similar arguments to Langmuir monolayers of octadecanol, we find at $30^{\circ} \mathrm{C}$, with the values $\eta=0.7$ and $K=3.45 \mathrm{~N} / \mathrm{m}$ determined in Sec. III, $\mu / K \approx 0.03$. Even if the decrease of the compression modulus $K$ on decreasing temperature (Fig. 9, right) is not taken into account, one finds that the ratio $\mu / K$ decreases to 0.007 close to the transition $L S-S$ due to an increase of $\eta$. Thus, such an interpretation of the exponent $\eta$ leads to extremely small values of the shear modulus, while the nature of the temperature dependence of $\eta$ (as well as the nature of the characteristic length $L$ and its temperature dependence), Fig. 6, remains unexplained.

The relevance - evident from Fig. 6 - of the phase transition $L S-S$ to the temperature dependence of the parameters $\eta$ and $L$ suggests the order parameter fluctuations close to the transition as the most plausible source of the broadening of the x-ray scattering peaks. Fluctuations with the structure of the backbone-ordered $S$ phase cause elastic distortions in the surrounding matrix (Fig. 1), since the centered rectangular unit cell of the $S$ phase differs from the hexagonal unit cell of the phase $L S$ by a rather large distortion (Fig. 5). Two symmetrically distinct order parameters may potentially describe the transition $L S-S[3,41,34]$ : the lattice distortion itself and the herringbone order parameter, which gives rise to distortions as a secondary effect at the transition. Although the first type of order parameter was rejected in the Landau theory $[41,3]$ because it cannot explain the tilt and distortion azimuths for the tilting phase transitions from $L S$ and $S$ phases simultaneously, we consider below the correlation functions for both order parameters. The difference between them concerns the temperature dependence of the parameters $\eta$ and $L$, rather than the shape of the correlation function $G(x)$.

The change of the unit cell shape caused by either of the possible order parameters can be described $[41,3]$ by the symmetric traceless tensor $\hat{\mathbf{u}}^{0}$. It is convenient to introduce the amplitude of the distortion $v$ and represent this tensor as $v \hat{\mathbf{u}}^{0}$, where $\operatorname{det}\left(\hat{\mathbf{u}}^{0}\right)=-1, \operatorname{trace}\left(\hat{\mathbf{u}}^{0}\right)=0$. The free energy of the distortions $F_{d}=-\int v c_{i j l m} u_{i j} u_{l m}^{0} d^{2} r$ caused by the internal strains $v \hat{\mathbf{u}}^{0}$ can be rewritten as

$$
F_{d}=-\Omega \sum_{\mathbf{k}} k P_{i} u_{i \mathbf{k}} v_{\mathbf{k}}^{*}
$$

where $P_{i}=c_{i j l m} n_{j} u_{l m}^{0}$ depends on the orientation of $\mathbf{k}$ but not on its length. Displacements $\mathbf{u}_{\mathbf{k}}$ are now the result of the fluctuation $v_{\mathbf{k}}$ : minimization of Eqs. (10) and (13) gives $u_{i \mathbf{k}}=k^{-1} C_{i j}^{-1} P_{j} v_{\mathbf{k}}$ and hence $\left\langle\left|\mathbf{Q}_{0} \cdot \mathbf{u}_{\mathbf{k}}\right|^{2}\right\rangle$ $=k_{\mathrm{B}} T\left(\mathbf{Q}_{0} \cdot \hat{\mathbf{C}}^{-1} \cdot \mathbf{P}\right)^{2}\left\langle\left|v_{\mathbf{k}}\right|^{2}\right\rangle / \Omega k^{2}$.

When the distortions are considered as the order parameter of the transition, the spectrum of harmonic fluctuations is [1] $\left\langle\left|v_{\mathbf{k}}\right|^{2}\right\rangle=w /\left(1+\xi^{2} k^{2}\right)$ with the temperature-dependent amplitude $w$ and the correlation length of fluctuations $\xi$. Both quantities $w$ and $\xi$ increase at the transition. If the amplitude of the herringbone wave $\varphi$ is considered as the order parameter of the transition, the distortion is a secondary effect in sense that $v \propto \varphi^{2}$ and hence $\left\langle\left|v_{\mathbf{k}}\right|^{2}\right\rangle=w^{2} /(1$ $\left.+\xi^{2} k^{2}\right)^{2}$. Then Eq. (9) can be written as

$$
U(r)=\eta \int \frac{d k}{k}\left[1-J_{0}(k r)\right] \frac{1}{\left(1+\xi^{2} k^{2}\right)^{m}},
$$

where $\eta=k_{\mathrm{B}} T w^{m} \overline{\left(\mathbf{Q}_{0} \cdot \hat{\mathbf{C}}^{-1} \cdot \mathbf{P}\right)^{2}} / 2 \pi$ and $m=1$ or 2 depending on the quantity considered as the order parameter of the transition, the distortion or the herringbone order parameter respectively. Close to the backbone-ordering transition, $\eta$ depends on temperature as $\eta \propto w^{m}$ and its increase is not restricted by any universal limit. Equation (14) gives rise to $U(r)=\eta \ln (r / \xi)$ on distances $r \gg \xi$. The correlation length of fluctuations $\xi$ limits the algebraic decay at small $r$ and thus replaces the largest wave vector in Eq. (11) by a temperature-dependent quantity.

Figure 11 compares the correlation functions $G_{\mathbf{Q}_{0}}(x)$ $=\exp [-U(x)]$ calculated using Eq. (14) $(m=1,2)$ with the analytical interpolation formula (6). When, in Eq. (6), the length $L$ is chosen equal to $1.15 \xi$ in the case of $m=1$ and 


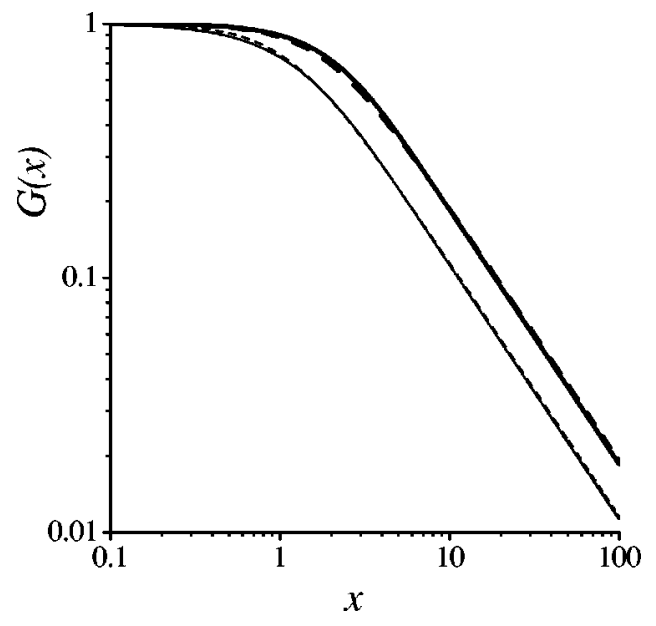

FIG. 11. Comparison of the correlation functions given by Eq. (14) with the analytical interpolation formula (6), $\eta=1$. Thin solid line: Eq. (14) with $m=1$. Thin dashed line: Eq. (6) with $L$ $=1.15 \xi$. Thick solid line: Eq. (14) with $m=2$. Thick dashed line: Eq. (6) with $L=1.95 \xi$.

equal to $1.95 \xi$ in the case of $m=2$, the difference between corresponding integrals and Eq. (6) becomes negligible. Therefore, Eq. (6) is a convenient interpolation formula which can be safely used to fit the experimental data instead of Eq. (14). One can conclude also that the shapes of the correlation functions for two possible order parameters discussed above are not distinguishable. The two order parameters differ in predictions of the temperature dependence of the exponent $\eta$. One expects $\eta \propto w^{2}$ when the transition is described by the herringbone order parameter and $\eta^{\propto} w$ when the distortion of the unit cell itself can be treated as the order parameter. The prediction of the Landau theory is $w$ $\propto\left(T-T_{0}\right)^{-1}$ and $\xi \propto\left(T-T_{0}\right)^{-1 / 2}$, where, for a second-order transition, the temperature $T_{0}$ is equal to the transition temperature but lower than it in the case of a first-order transition. Hence, fluctuations increase at the first-order transition but remain finite. The qualitative picture given above will not change when one goes beyond Landau theory, while the temperature dependencies of the parameters $\eta$ and $\xi$ change.

\section{CONCLUSIONS}

The present $\mathrm{x}$-ray diffraction study of octadecanol monolayers has concentrated on measurement and analysis of the peak profiles. We found significant peak broadening close to the first-order $L S-S$ phase transition. The peaks become sharper on cooling in the $S$ phase and on heating in the $L S$ phase. Comparison of monolayers prepared in different ways showed that the peak profiles do not depend on monolayer history and hence the observed peak shapes are descriptive of the thermal equilibrium properties of the system.

We have determined the correlation functions of positional order in Langmuir monolayers of octadecanol directly from the profiles of the measured x-ray diffraction peaks, without introducing any model in advance. This was possible because the monolayer is a two-dimensional powder of randomly oriented domains. The average of the structure factor over domain orientations is equivalent to a cut in the corre- lation function in the direction of the relevant reciprocallattice vector.

We found algebraic decay of positional correlations, $G(x) \propto x^{-\eta}$, with the temperature-dependent exponent $\eta$. The values of $\eta$ vary from $\eta \approx 0.5$ far from the backboneordering phase transition $L S-S$ to approximately 3 close to it. Even the smallest values of the exponent $\eta$ found in the present study exceed the limit $\eta<1 / 3$ imposed by the dislocation-mediated melting theory for the exponent in the solid phase before the melting transition.

A sharp increase of the exponent at the transition $L S-S$ points to backbone ordering as a source of the additional positional disorder in the system. We attribute the unusually broad peaks to elastic distortions caused by fluctuations (with the structure of the new phase) in the surrounding matrix. They do not destroy the quasi-long-range positional order but cause local strains that give rise to an additional contribution to the exponent $\eta$.

The algebraic decay is restricted, at small distances, by a length $L$, which also increases close to the transition. This length can be treated as the correlation length of the order parameter fluctuations. At the temperatures investigated in the present experiment (i.e., not less than $0.5^{\circ} \mathrm{C}$ from the transition) it remains in the range of several lattice spacings, which explains the absence of superstructure peaks in the $L S$ phase at positions corresponding to the structure of the $S$ phase. There is no evidence for a characteristic length (e.g., a crystallite size) that would restrict the algebraic decay at large distances. The algebraic decay of positional correlations points to quasi-long-range order on the available length scale in both phases $L S$ and $S$. High-resolution diffraction measurements that are possible at third generation synchrotron sources could extend the distance range by at least 50 times [7].

Based on the widths of the peaks that exceed the resolution limit, the phases $L S$ and $S$ have often been considered as hexatics. Sirota [34] pointed out that, since we are in regimes where a hexatic and a crystal cannot necessarily be distinguished experimentally, theories of phase transitions should not be too sensitive to such a distinction. The Landau theory of phase transitions in Langmuir monolayers was initially formulated in terms of phase transitions between 2Dcrystalline phases [41] and then reformulated in terms of hexatic phases [3] using essentially the same free energy expansions. Hence, it complies with this demand. However, our considering the $S$ phase as a 2D solid raises a question of the nature of the $S-C S$ transition, which was treated [3] as a crystallization of the mesophase. A study of the structural difference between $S$ and $C S$ phases in a high-resolution experiment, as well as a detailed study of positional order in the tilted phases, may demand further development of the Landau theory of phase transitions in Langmuir monolayers.

\section{ACKNOWLEDGMENTS}

We are grateful to HASYLAB (Hamburg, Germany) for beam time. V.M.K. thanks Efim Kats for helpful discussions. This work was supported by the Alexander von Humboldt Stiftung (V.M.K.), Deutsche Forschungsgemeinschaft (G.B.), the Danish Dansync, and the E.U.'s TMR program (P.B.H. and K.K.). 
[1] L. D. Landau and E. M. Lifshitz, Statistical Physics: Pt. 1 (Pergamon, Oxford, 1980).

[2] D. R. Nelson and B. I. Halperin, Phys. Rev. B 19, 2457 (1979).

[3] V. M. Kaganer and E. B. Loginov, Phys. Rev. Lett. 71, 2599 (1993); Phys. Rev. E 51, 2237 (1995).

[4] J. Als-Nielsen, D. Jacquemain, K. Kjaer, F. Leveiller, M. Lahav, and L. Leiserowitz, Phys. Rep. 246, 251 (1994).

[5] I. Weissbuch, R. Popovitz-Biro, M. Lahav, L. Leiserowitz, K. Kjaer, and J. Als-Nielsen, Adv. Chem. Phys. 102, 39 (1997).

[6] K. Kjaer, Physica B 198, 100 (1994).

[7] C. Zakri, A. Renault, J.-P. Rieu, M. Vallde, and B. Berge, Phys. Rev. B 55, 14163 (1997).

[8] W. D. Harkins and L. E. Copeland, J. Chem. Phys. 10, 242 (1942).

[9] G. T. Barnes and D. S. Hunter, J. Colloid Interface Sci. 136, 198 (1990).

[10] G. A. Lawrie and G. T. Barnes, J. Colloid Interface Sci. 162, 36 (1994).

[11] C. Lautz, Th. M. Fischer, and J. Kildea, J. Chem. Phys. 106, 7448 (1997).

[12] G. Brezesinski, V. M. Kaganer, H. Möhwald, and P. B. Howes, J. Chem. Phys. 109, 2006 (1998).

[13] C. Lautz, T. M. Fischer, M. Weygand, M. Lösche, P. B. Howes, and K. Kjaer, J. Chem. Phys. 108, 4640 (1998).

[14] J. Als Nielsen and A. K. Freund, Rev. Sci. Instrum. 63, 1156 (1992).

[15] S. Riviére, S. Hénon, J. Meunier, D. K. Schwartz, M.-W. Tsao, and C. M. Knobler, J. Chem. Phys. 101, 10045 (1994).

[16] B. E. Warren and B. L. Averbach, J. Appl. Phys. 21, 595 (1950).

[17] B. E. Warren, X-Ray Diffraction (Addison-Wesley, Reading, Massachusetts, 1969).

[18] H. P. Klug and L. E. Alexander, X-Ray Diffraction Procedures For Polycrystalline and Amorphous Materials, 2nd ed. (Wiley, New York, 1974).

[19] M. A. Krivoglaz, X-Ray and Neutron Diffraction in Nonideal Crystals (Springer, Berlin, 1996).

[20] For the case of more than one molecule per unit cell, read "unit cell structure factor" instead of "molecular structure factor.',
[21] W. Marshall and S. Lovesey, Theory of Thermal Neutron Scattering. The Use of Neutrons for the Investigation of Condensed Matter (Oxford University Press, London, 1971).

[22] V. M. Kaganer, B. I. Ostrovskii, and W. de Jeu, Phys. Rev. A 44, 8158 (1991).

[23] The possible inequivalence of the two molecules in the herringbone-ordered structure $[24,4]$ is not relevant here.

[24] J.-L. Wang, F. Leveiller, D. Jacquemain, K. Kjaer, J. AlsNielsen, M. Lahav, and L. Leiserowitz, J. Am. Chem. Soc. 116, 1192 (1994).

[25] M. C. Shih, T. M. Bohanon, J. M. Mikrut, P. Zschack, and P. Dutta, Phys. Rev. A 45, 5734 (1992).

[26] B. Lin, J. B. Peng, J. B. Ketterson, P. Dutta, B. N. Thomas, J. Buontempt, and S. A. Rice, J. Chem. Phys. 90, 2393 (1989).

[27] A. M. Bibo and I. R. Peterson, Adv. Mater. 2, 309 (1990).

[28] E. I. Kats and J. Lajzerowicz, Zh. Éksp. Teor. Fiz. 110, 899 (1996) [JETP 83, 495 (1996)].

[29] V. M. Kaganer, I. R. Peterson, R. M. Kenn, M. C. Shih, M. Durbin, and P. Dutta, J. Chem. Phys. 102, 9412 (1995).

[30] C. Fradin, J. Daillant, A. Braslau, D. Luzet, M. Alba, and M. Goldmann, Eur. Phys. J. B 1, 57 (1998).

[31] K. Kjaer, J. Als-Nielsen, C. A. Helm, P. Tippman-Krayer, and H. Möhwald, J. Phys. Chem. 93, 3200 (1989).

[32] These measurements were performed at the Troika II beamline at ESRF, Grenoble. We thank Detlef Smilgies and Nathalie Boudet for setting up the experiment.

[33] E. B. Sirota, D. M. Singer, and H. E. King, J. Chem. Phys. 100, 1542 (1994).

[34] E. B. Sirota, Langmuir 13, 3849 (1997).

[35] E. B. Sirota, H. E. King, H. Shao, and D. M. Singer, J. Phys. Chem. 99, 798 (1995).

[36] I. R. Peterson and R. M. Kenn, Langmuir 10, 4645 (1994).

[37] L. E. Copeland, W. D. Harkins, and G. E. Boyd, J. Chem. Phys. 10, 357 (1942).

[38] B. Jancovici, Phys. Rev. Lett. 19, 22 (1967).

[39] Y. Imry and L. Gunther, Phys. Rev. B 3, 3939 (1971).

[40] F. Weling and A. Griffin, Phys. Rev. B 25, 2450 (1982).

[41] V. M. Kaganer and V. L. Indenbom, J. Phys. II 3, 813 (1993). 